\title{
Pitting failure of helical gears induced by trochoidal interference and multidirectional, interacting wear
}

\author{
Koji KUMAGAI*, Yuta NAITO* and Syuhei KUROKAWA** \\ *Nissan Motor Co., Ltd \\ 560-2 Okatsukoku, Atsugi, Kanagawa 243-0192, Japan \\ E-mail:ku-kumagai@mail.nissan.co.jp \\ **Department of Mechanical Engineering, Kyushu University \\ 744 Motooka, Nishi-ku, Fukuoka 819-0395, Japan
}

Received: 28 February 2020; Revised: 12 April 2020; Accepted: 12 May 2020

\begin{abstract}
Improving gear mesh efficiency contributes to reducing the environmental impact of vehicles. This study examined the effect of adjusting gear specifications on improving mesh efficiency. When the gear ratio of cylindrical gears is relatively high, it tends to increase the sliding velocity between the engaging gear teeth. Moving the contact region toward the base circle of the pinion along the line of action is considered to be one solution for reducing friction loss. However, it may cause deterioration of tooth surface strength because the radius of curvature of the tooth surface becomes smaller on account of being closer to the base circle. In order to confirm the influence of the gear meshing region on tooth surface strength, gear durability tests were carried out and tooth damage states were observed in detail under a microscope. The results revealed that pitting failure occurred near the tooth root region of the pinion. Furthermore, trochoidal interference due to the high torque condition extended over the base circle. In this case, trochoidal interference has a greater influence on pitting failure. In order to investigate the effect of reducing trochoidal interference near the base circle on pitting failure, further gear durability tests were conducted using the mating gears with large tooth tip modification. However, the effect on improving pitting durability was limited. Multidirectional, interacting wear was observed not only in the profile direction due to trochoidal interference but also in the lead direction due to edge contact. Such wear changed the location of pits. The results of these detailed investigation revealed a new viewpoint for explaining the mechanism of these phenomena in terms of conflict between pitting and multidirectional, interacting wear. Presumably, pitting failure changes if the state of progression of trochoidal interference and wear in the lead direction changes. In order to confirm the influence of such wear on pitting durability, a gear durability test was conducted using gears with tooth tip modification and endface relief. The test results confirmed that the location of pits and pitting durability changed. These results revealed that pitting durability and the state of damage were influenced sensitively and compositely by multidirectional, interacting wear, i.e., wear in the profile and lead directions.
\end{abstract}

Keywords : Gear, Machine element, Automobile, Pitting, Trochoidal interference, Base circle, Tip modification, Multidirectional interacting wear, Edge contact

\section{Introduction}

Gears are power transmission elements that are widely used in industrial machines for obtaining high efficiency. In recent years, further energy saving has been demanded of automobiles. Power loss reduction is strongly required for gears used in automotive transmissions and reducers of electric vehicles (EVs). The power loss of gears includes churning loss of the lubricant and gear meshing loss. The reducer of electric vehicles has a relatively small number of parts, so gear meshing loss accounts for more than half of the total power loss depending on the operating conditions.

There are some measures for reducing gear meshing loss. Attention has been paid to the gear finishing process in which gear meshing loss can be reduced by improving gear tooth surface roughness (Kumagai, et al., 2017), (Okamoto 
et al, 2017). Another approach to reducing gear meshing loss is to devise better gear specifications. For example, there is a report that an asymmetric gear with an action tooth surface having a high-pressure angle for reducing surface contact stress improves oil film formation between tooth surfaces and thereby reduces meshing loss (Okumura, et al., 2017).

In addition, to reduce the sliding speed of tooth pairs, there is a report that changing the gear meshing range on the line of action leads to a reduction of gear meshing loss. Cylindrical gears used for the final reduction gear pair in automotive transmissions and reducers of EVs have a relatively high gear ratio. Because of their geometric properties, the gear meshing range of the tooth dedendum is apt to become large. That tends to increase the gear meshing region away from the pitch point, leading to an increase in gear meshing loss due to the higher sliding velocity. There is a method of shortening the gear meshing length by means of a low tooth depth in order to suppress the increased loss. However, it reduces the gear contact ratio and causes greater gear noise. In order to suppress that influence and reduce meshing loss, the integral value of the sliding velocity of the gear meshing region is reduced as the range of gear meshing approaches the tooth root of the small pinion (Morikawa, et al., 2012). However, if the contact region extends close to the base circle of the pinion, it affects tooth surface strength.

Consequently, it is difficult to balance gear meshing with other performance attributes at a high level. It is necessary to clarify the relationships among contradicting performance attributes to achieve it.

It is generally known that tooth surface strength is influenced by trochoidal interference (Komori, et al., 2004), (Yoshizaki, 2016) in which the tooth tip edge comes in contact with the mating tooth root region. However, at present a method has not been established for accurately estimating gear strength that takes into consideration trochoidal interference.

In this study, attention was paid to the influence on tooth surface strength, thought to be contradictory to the improvement of gear mesh efficiency by changing the gear meshing region on the line of action. Gear strength and durability tests were conducted to investigate the state of tooth surface damage. The results showed that pitting damage and trochoidal interference occurred in the tooth root region of the pinion like that described in previous reports (Komori, et al, 2004), (Yoshizaki, 2016). Detailed observation of the state of tooth surface damage under a microscope, revealed that trochoidal interference went beyond the base circle of the pinion to the tooth bottom and that gouging occurred on the tooth surface at the position of interference.

In order to investigate the influence on suppressing a decline in tooth surface strength by mitigating trochoidal interference near the base circle of the pinion, durability tests were conducted using mating gears having large tooth tip modification. The results showed that trochoidal interference was avoided near the base circle as intended. However, pitting durability did not change appreciably, and the effect on suppressing the decline in tooth surface strength was limited. Observation of the tooth surface indicated that tooth surface pitting was influenced not only by trochoidal interference but also by wear that started from edge contact in the lead direction. The results suggested that pitting durability and the state of damage are influenced sensitively and compositely by multidirectional, interacting wear, i. e., wear in the profile and lead directions. These results are described in detail in subsequent section.

In this paper, the word wear is defined as a phenomenon whereby fine wear powder is removed from the surface to cause successive weight loss and it is distinguished from pitting. Although trochoidal interference is the term that has often been used heretofore for interference between the pinion and the internal gear, the term is used here in a broader sense as indicating interference between meshing external teeth except in the position of meshing with the involute gear.

\section{Effect of adjusting gear specifications on reducing gear meshing loss}

Figure 1 is a schematic of the relationship between the sliding velocity distribution and the contact region on the line of action of the gear pair. First, we will consider the contact region indicated by the blue line on the line of action. When the gear ratio of a cylindrical gear is relatively high, the length of the approach path decreases and the length of the recess path increases, and the region distant from the pitch point increases. As a result, the integral of the sliding velocity on the recess contact side increases. The gear meshing region is moved in the direction of the red line in the figure to reduce gear meshing loss, without changing the length of the path of contact. As the ratio of the length between the approach path and recess path approaches one, mesh efficiency increases.

Mesh efficiency was calculated using Niemann's equation (Niemann, 1968) expressed as Eq. (1) in order to understand the concrete relationship with the meshing region. The meshing region on the line of action is moved by means of an addendum modification coefficient as a variable. The basic gear specifications and gear finishing process 


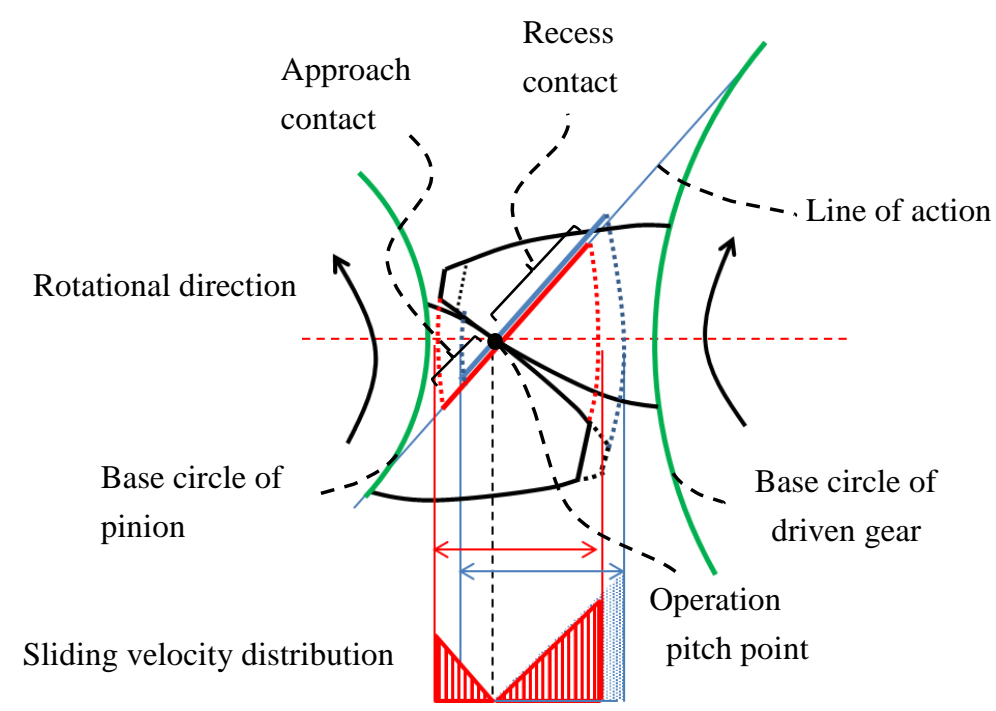

Fig. 1 Schematic of sliding velocity distribution of cylindrical gear pair for a relatively high gear ratio. Approach contact ratio and recess contact ratio tend to decrease and increase, respectively. When the contact range changes from the blue line to the red line, the integral value of the sliding velocity can be reduced.

$$
\eta=\left[1-\frac{\pi \mu}{\cos \beta}\left(\frac{1}{z_{1}}+\frac{1}{z_{2}}\right)\left(1-\varepsilon+\varepsilon_{1}{ }^{2}+\varepsilon_{2}{ }^{2}\right)\right]
$$

are shown in Table 1. The notations $z_{1}$ and $z_{2}$ are the number of teeth of the pinion and driven gear, respectively. $\beta$ is the helix angle, $\varepsilon_{1}$ is the ratio of the approach path, $\varepsilon_{2}$ is the ratio of the recess path, $\varepsilon=\varepsilon_{1}+\varepsilon_{2}$ is the profile contact ratio, and $\mu$ is the friction coefficient.

Table 1 Basic gear specifications and gear finishing process.

\begin{tabular}{|c|c|}
\hline Number of teeth & $17 / 74$ \\
\hline Module & 2.25 \\
\hline Pressure angle & $17.5^{\circ}$ \\
\hline Helix angle & $31.5^{\circ}$ \\
\hline Center distance & $120.4 \mathrm{~mm}$ \\
\hline \hline $\begin{array}{c}\text { Gear finishing process } \\
\text { (Pinion / Driven gear) }\end{array}$ & Gear honing / Gear generate grinding \\
\hline
\end{tabular}

Figure 2 shows the calculation results for $\eta$. The horizontal axis represents the ratio between the approach path and

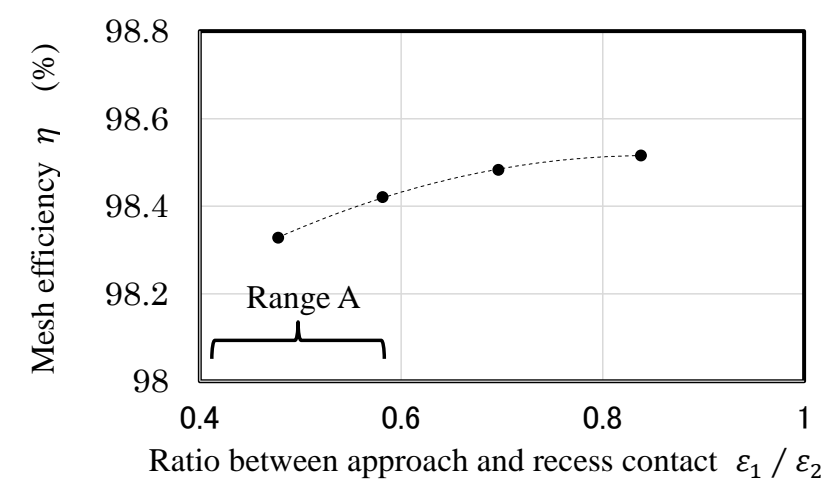

Fig. 2 Calculation results for mesh efficiency $\eta$ according to the contact ratio between approach contact $\varepsilon_{1}$ and recess contact $\varepsilon_{2}$. Range A indicates the value of ordinary gear pairs applied to the final reduction gear of automotive transmissions or EVs reducers. $\eta$ improves so that $\varepsilon_{1} / \varepsilon_{2}$ approaches one. 
recess path $\varepsilon_{1} / \varepsilon_{2}$, and the value of $\mu$ is 0.07 . The value of $\varepsilon_{1} / \varepsilon_{2}$ of the gear pair used for the final reduction gear pair in automotive transmissions and reducers for EVs is often in the range indicated by $\mathrm{A}$ in the figure. As $\varepsilon_{1} / \varepsilon_{2}$ approaches one, mesh efficiency increases.

However, since the contact region extends close to the base circle of the pinion, the relative radius of curvature $R_{e}$ becomes small. Figure. 3 shows the relation between $R_{e}$ and $\varepsilon_{1} / \varepsilon_{2}$ at the mesh starting point of tooth root side of the pinion. The value of vertical axis of right side in the figure represents the maximum contact stress in the gear tooth surface when input torque is $600 \mathrm{Nm}$. Reducing $R_{e}$ leads to increase the maximum contact stress. This causes a reduction of the tooth surface strength.

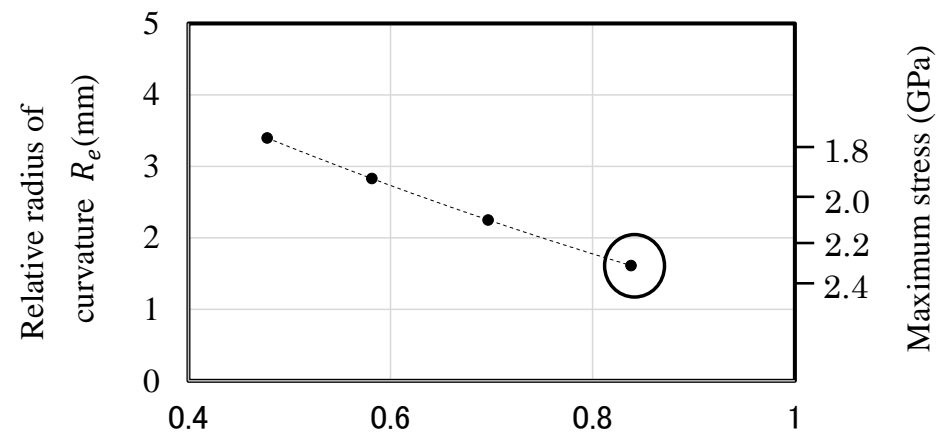

Ratio between approach and recess contact $\varepsilon_{1} / \varepsilon_{2}$

Fig. 3 Equivalent radius of curvature $R_{e}$ according to the contact ratio between approach contact $\varepsilon_{1}$ and recess contact $\varepsilon_{2}$. The horizontal axis corresponds to Fig. 2 . As $R_{e}$ nears zero, $\varepsilon_{1} / \varepsilon_{2}$ approaches one. The value of vertical axis of right side represents the maximum contact stress in the gear tooth surface when input torque is $600 \mathrm{Nm}$. Reducing $R_{e}$ leads to increase the maximum contact stress. This causes a reduction of the tooth surface strength. The circle described by solid line refers to Fig.4.

The load distribution on the simultaneous contact line was calculated using Kubo and Umezawa's method (Kubo, et al., 1977) for the gear pair in the circle in the figure. The calculation results for the distribution of the tooth contact stress are given in Fig. 4. The symbol $\mathrm{X}$ indicates the position of maximum contact stress. Contact stress is shown in different colors for values obtained by dividing the maximum contact stress into 10 equal parts. Because $R_{e}$ becomes small on the root side of the pinion, contact stress increases and a possible decline in tooth surface strength is a matter of concern.

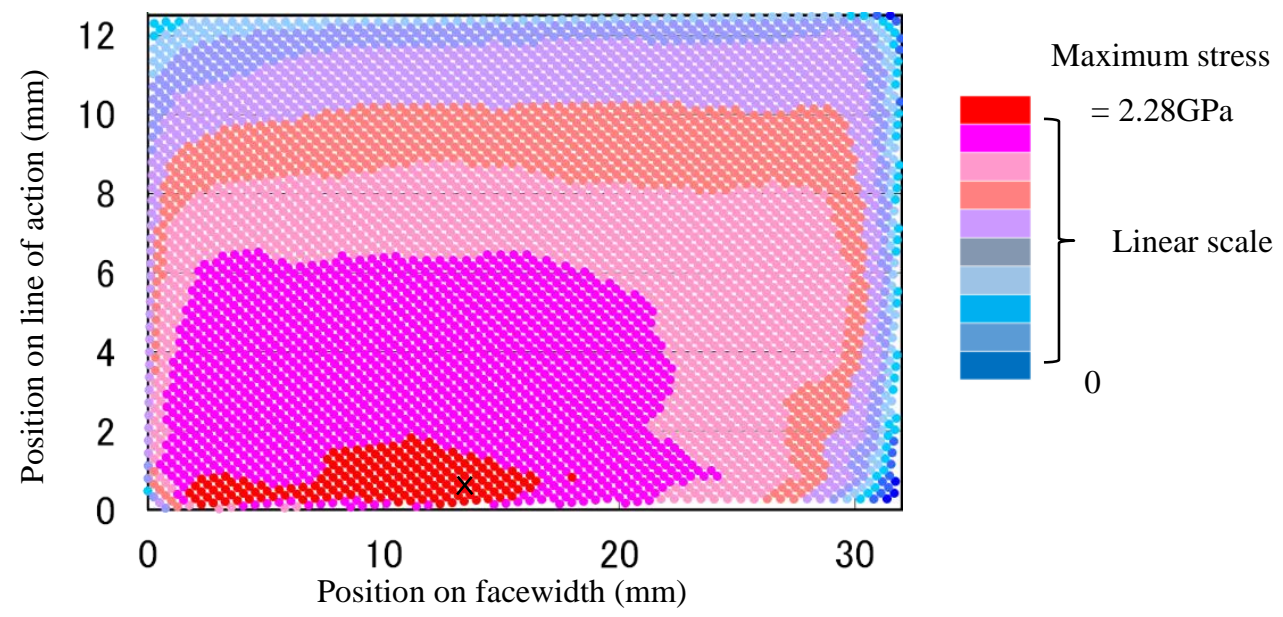

Fig. 4 Calculation results for contact stress distribution of the gear specification in the circle described by solid line in Fig. 3. The symbol $\mathrm{X}$ is the position of maximum contact stress. The color map is expressed by dividing the value of the maximum contact stress into 10 equal parts. The value of contact stress of the tooth root area of the pinion is large due to the small equivalent radius of curvature.

As mentioned above, when gear mesh efficiency is improved by devising better gear specifications, it is considered to be contradictory to gear surface strength. The following section describes the gear durability tests that were conducted to confirm the influence on tooth surface strength. 


\section{Influence of adjusting gear specifications on tooth surface damage}

Gear durability tests were carried out to investigate the influence on the state of tooth surface damage when the gear meshing region reached near the base circle of the pinion. Figure 5 represents the measured tooth flank error of the pinion and driven gear. The gears had no large amount of tooth tip modifications. Table 2 shows the experimental conditions.

Table 2 Lubricant and gear durability test conditions.

\begin{tabular}{|c|c|}
\hline Lubrication type & ATF (Bath lubrication) \\
\hline \multirow{2}{*}{ Oil kinematic viscosity } & $5.738 \mathrm{~mm}^{2} / \mathrm{s}\left(100^{\circ} \mathrm{C}\right)$ \\
\cline { 2 - 2 } & $26.95 \mathrm{~mm}^{2} / \mathrm{s}\left(40^{\circ} \mathrm{C}\right)$ \\
\hline Supply oil temperature & $100{ }^{\circ} \mathrm{C}$ \\
\hline Pinion torque & $600 \mathrm{Nm}$ \\
\hline Pinion rotational speed & $2000 \mathrm{~min}^{-1}$ \\
\hline
\end{tabular}

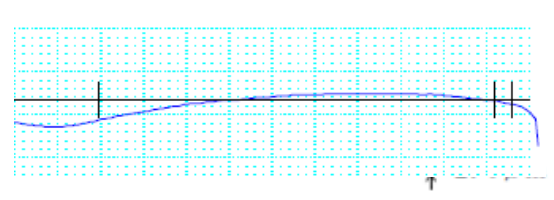

(a) Measured profile of pinion

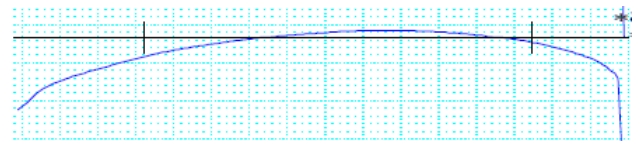

(c) Measured helix of pinion

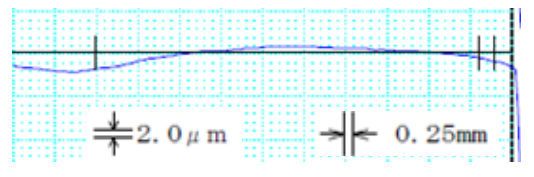

(b) Measured profile of driven gear

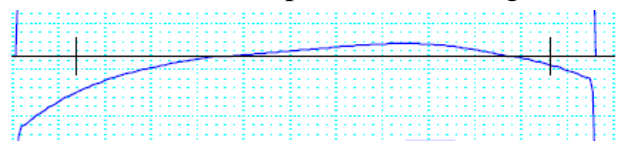

(d) Measured helix of driven gear

Fig. 5 Measured profile and helix of pinion and gear. The gears have no large tooth tip modifications.

Figure 6 presents the results of the gear durability test. The red circle shows the result of the gear pair described in Fig. 5 . The black circles show the results of the test as for database before than that of the red circle. Pitting durability of red circle was reduced to half of the estimated level as expected from database. The process of gears was same and it was considered that the pitting durability was similar. However, the difference of the durability was large. It is considered an unknown factor hides behind. The cause of the difference was thought to be the slight change in the tooth surface contact state and trochoidal interference, but it was not clear. It is important to suppress the dispersion of pitting durability for gears in the automobile. The tooth surface failure state was then observed using a microscope in order to investigate trochoidal interference in detail.

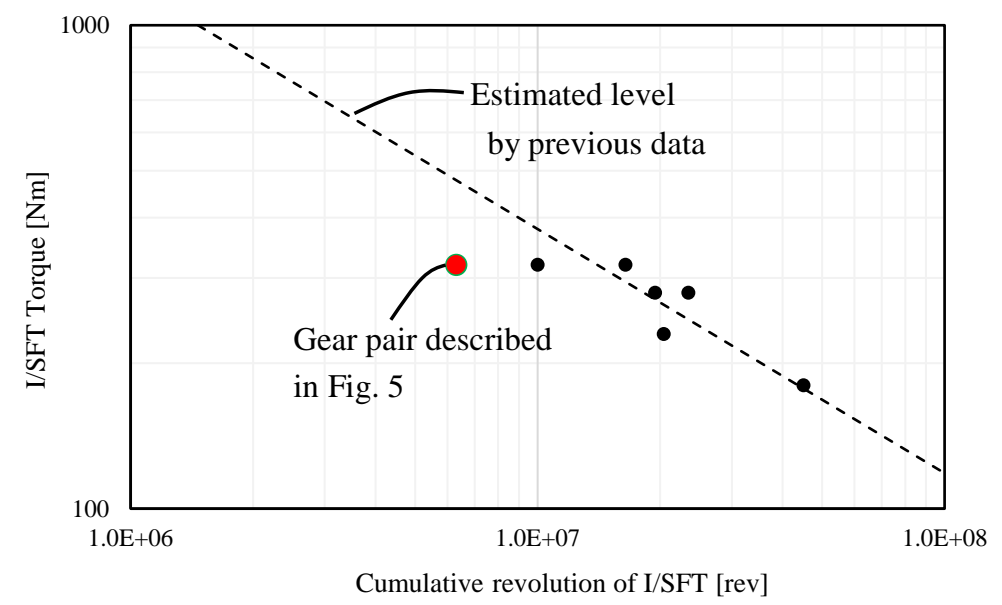

Fig. 6 Results of pitting durability test. Pitting durability was $6.3 \times 10^{6}$ cycles and was reduced by half compared with before. 
Figures 7 and 8 present the tooth damage state of the pinion and the driven gear after the durability test, respectively. These photos are replicas of the teeth that were taken using a microscope, VHX-5000 by Keyence. The magnification was used up to 200 times in this study. Observation of the photos revealed several pits in the gear mesh start region in Fig. 7. The tooth surface damage was not seen clearly in Fig. 8, but when the image was enlarged near the tooth tip on the gear mesh start side as shown in Fig. 9, traces of strong mesh contact can be observed from the tooth tip toward the tooth surface. Presumably, the tooth tip of the gear came in strong contact with the tooth root of the pinion, resulting in pitting.

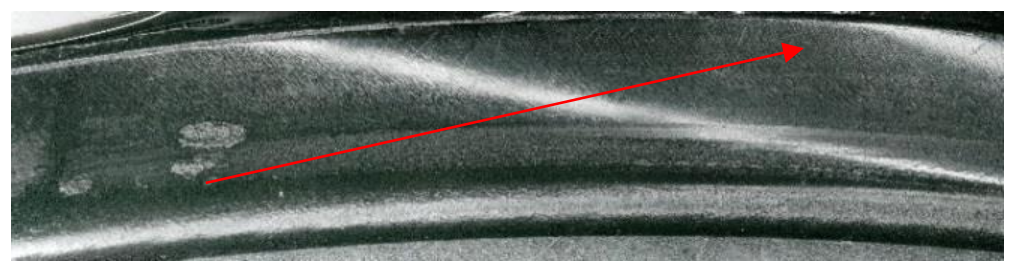

$\mathrm{N}=6.3 \times 10^{6}$

cycles

Fig. 7 Damage on the pinion tooth surface after the gear durability test. Arrow indicates the gear meshing direction. Some pits occurred near the meshing start region.

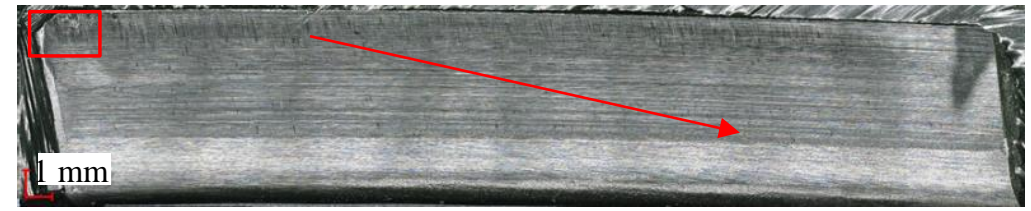

Fig. 8 Damage on the driven gear tooth surface after the gear durability test. Arrow indicates the gear meshing direction. The square in red are referred by Fig. 9. No visible damage occurred.

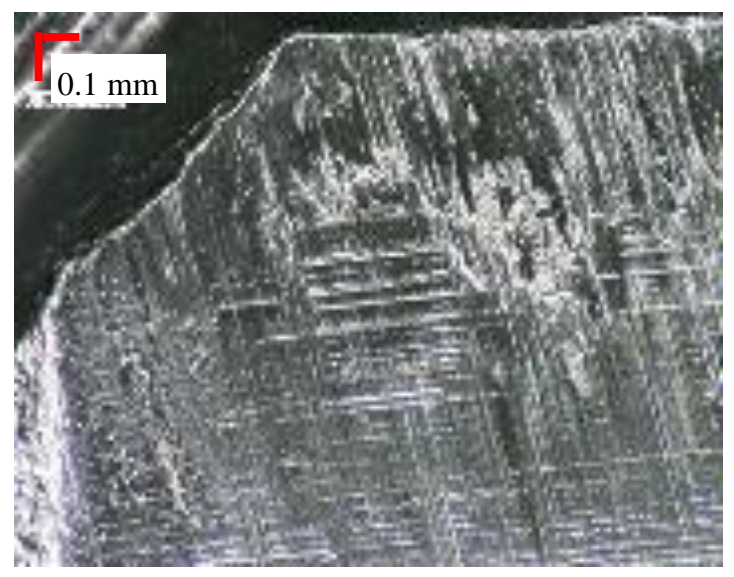

Fig. 9 Enlarged image near the meshing start region of the tooth tip which is shown in a red square in Fig. 8. Traces of strong mesh contact can be observed from the tooth tip toward the tooth surface.

In order to investigate the pitting state in Fig. 7 more closely, an observation was made of an enlarged image near the position of pits in Fig. 10. The diameter of the mesh start point calculated geometrically is denoted as $D_{S}$, the base circle diameter of the pinion as $D_{B}$ and the operating pitch diameter as $D_{P}$. The values of $D_{S}, D_{B}, D_{P}$ are $42.21 \mathrm{~mm}$, 42.08mm, 44.98mm respectively. The pits are labeled as A, B and C. Pit A occurred at the edge of the tooth width and was probably caused by strong edge contact. Pit B occurred starting from $D_{S}$ at a slightly remote position in the lead direction from pit A. Pit $\mathrm{C}$ probably grew on the addendum side starting from pit $\mathrm{B}$.

Figure 11 is a further enlarged view of Fig. 10 in order to observe the state of damage near the base circle in more detail. A lot of micropits thought to be the initial pits occurred on the addendum side of $D_{S}$, and pit B occurred a little on the addendum side of the micropits. In addition, the cutting region of the trochoidal interference (Kubo et al., 2019) was seen near $D_{S}$, and the region extended across $D_{B}$ toward the tooth bottom where a lot of sliding traces by gear tooth tip edge or abrasive powders can be seen. 


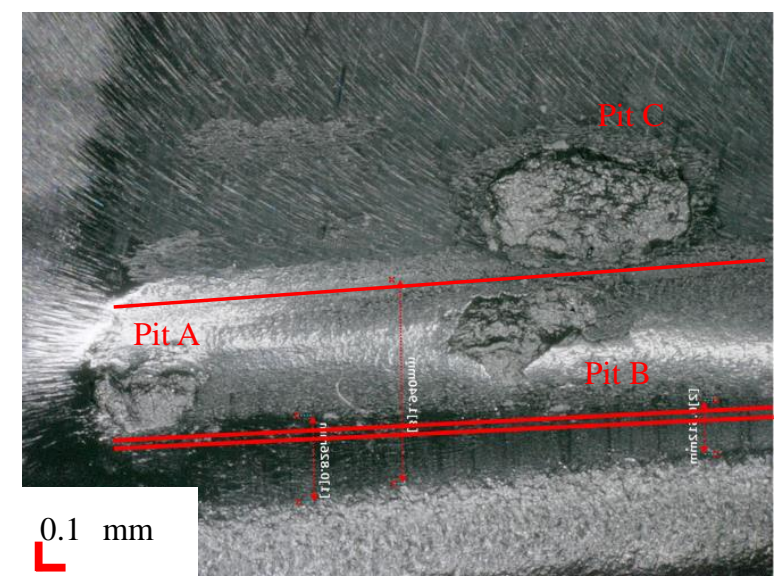

Operation pitch circle $D_{P}$

Start point of contact $D_{S}$

Base circle $D_{B}$

Fig. 10 Enlarged image of the lower left area in Fig. 7. The positions of the operation pitch circle, start point of contact and base circle are indicated.

Start point of contact $D_{S}$

Base circle $D_{B}$

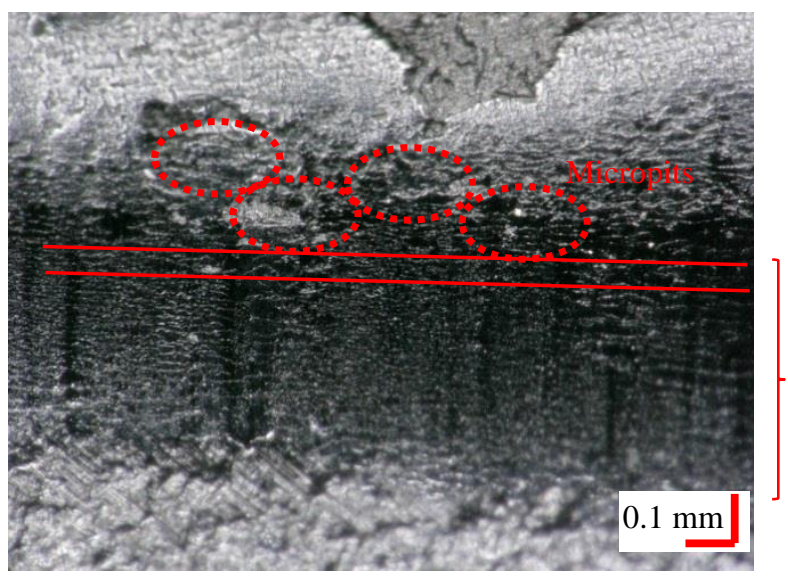

Concavity due to

trochoidal interference

Fig. 11 Further enlarged image of the tooth root region in Fig. 10. The positions of the Start point of contact, base circle and trochoidal interference are indicated.

There is a shoulder in the border of the cutting region. Because trochoidal interference caused high contact stress, the crack propagated from the shoulder, and presumably caused the pit. These damage results indicated that the tooth surface tended to suffer pitting failure probably because trochoidal interference occurred near $D_{S}$ and $D_{B}$ with a relatively small radius of curvature $R_{e}$. It is inferred that trochoidal interference had a greater influence on pitting.

The factor causing trochoidal interference to extend across $D_{S}$ and $D_{B}$ toward the tooth bottom is considered here. Trochoidal interference caused by bending deflection of a gear pair under a high load has generally occurred on the addendum side from $D_{S}$. However, the position of trochoidal interference in this study extended across $D_{S}$ toward the tooth bottom. Figure 12 presents the geometrical calculation results for the trochoidal trajectory of the tooth tip of the gear when the rotation of the gear is viewed in a fixed relative coordinate system. The red line shows the tooth crosssectional shape of the pinion and the trajectory in blue shows the tooth tip of the driven gear. $D_{S}, D_{P}$ and $D_{B}$ are shown together in the figure. The right-hand diagrams in Fig. 12 are enlarged representations near the tooth root of the pinion. It also shows the start point of contact $D_{S}$ with trajectory of gear tip. The position of the start point of contact $D_{S}$ represents the geometrical involute mesh start point on the line of action, and the trochoidal curve moves in the direction of the arrow as gear meshing progresses. Observation of the trajectory reveals that the tooth tip passes the position near the pinion tooth surface. This result suggests that the tooth tip of the driven gear came in contact with the tooth root of pinion due to its deformation under the high load condition. In fact, the concavity due to the trochoidal interference reached over the base circle by $0.3 \mathrm{~mm}$ to $0.4 \mathrm{~mm}$ in Fig. 11. In this study, the word trochoidal interference is used for the phenomenon that the tooth root is concaved by the tooth tip of the counter gear due to the deflection of a gear pair under a high load. 
Trochoidal trajectory of gear tip
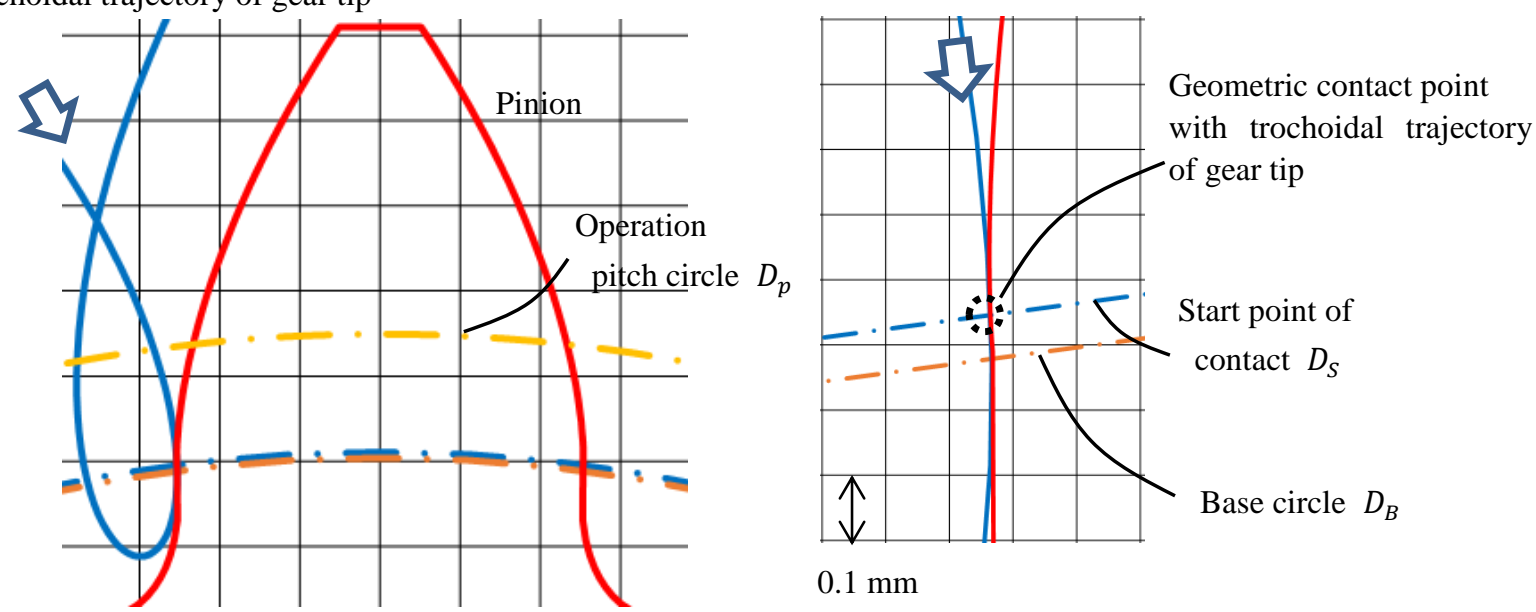

$0.1 \mathrm{~mm}$

Fig. 12 Representation of trochoidal trajectory of gear tip. Diameter of the start point of contact, operation pitch circle and base circle diameter are also indicated. Right-hand diagrams are enlarged representations near the tooth root of the pinion. When the gear pair deforms under the high load condtion, the tooth tip of the gear comes in contact with the tooth root beyond the base circle of the pinion easily.

Based on the results mentioned above, when the start point of involute meshing is close to the base circle as a result of adjusting the gear specifications, trochoidal interference might occur across or near the base circle of the pinion. It generates the shoulder which has a relatively small radius of curvature and has a greater influence on pitting damage. Also, the shoulder was formed near the region of maximum contact stress in Fig. 4. As a result, the micropits and the pits in Fig. 10 and Fig. 11 were generated due to the shoulder and the maximum contact stress.

The following section describes the gear durability tests that were conducted using mating gears with large tooth tip modification in order to investigate the effect of reducing trochoidal interference near the base circle on suppressing pitting failure.

\section{Variation in the location of pits and pitting durability due to multidirectional, interacting wear 4-1. Estimated mechanism of the variation in the location of pits and pitting durability}

Durability tests were conducted on a gear pair, denoted as A, having the same gear specifications described in Table. 1. The experimental conditions were the same as Table 2. Figure 13 shows the measured profile of Gear A which has large amount of tip modification in order to avoid trochoidal interference near the base circle of the pinion. The tip modification was processed by the gear generate grinding.

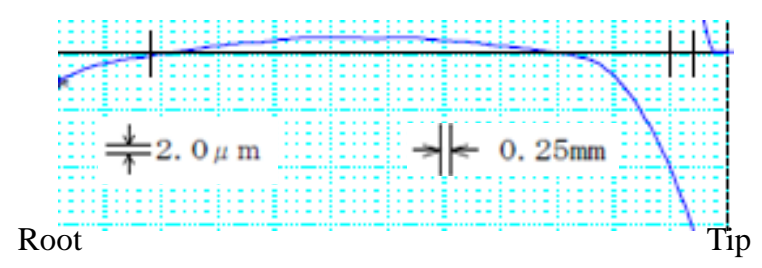

Profile of Gear A

Fig. 13 Measured profile of Gear A with large amount of tooth tip modification in order to decrease the trochoidal interference near base circle of pinion.

The durability test results indicated that a pit developed on gear pair A at loading cycles of $7.2 \times 10^{6}$. The tooth tip modification had a limited effect for gear pair A. It was assumed reducing trochoidal interference near the base circle would work to suppress the decline in pitting durability. However, there was virtually no change from the results described in section 2, and the occurrence of trochoidal interference near the base circle alone was not sufficient to explain the cause of this decline.

The tooth surface failure state was then investigated in detail in order to clarify the reason why pitting durability was 
not improved by mitigating trochoidal interference.

Photos of the tooth damage on pinion A are shown in Fig. 14. The pit on pinion A was located near the center of the tooth width.

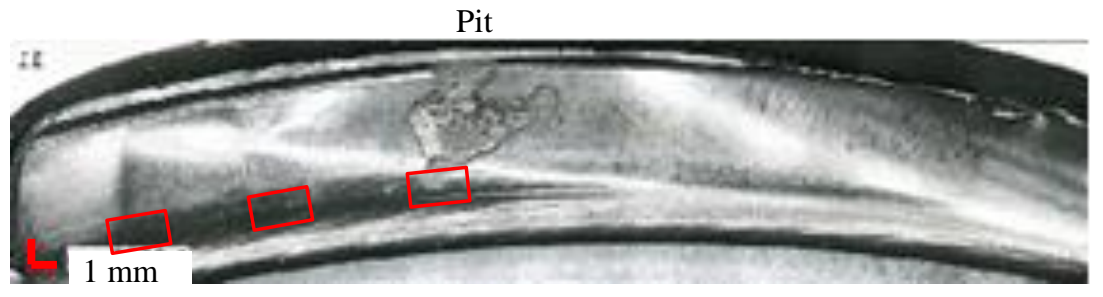

Pinion A, $7.2 \times 10^{6}$ cycles

Fig. 14 State of pinion tooth damage after durability tests. The square figures in red are referred by Fig. 15. Even for the same specifications, pitting durability and the location of pit differed.

Figure 15 shows enlarged images of the tooth root region in Fig. 14. Trochoidal interference was avoided across the start point of contact $D_{S}$ or the base circle because of the tooth tip modification of driven gear. However, pits occurred near the shoulder caused by trochoidal interference on the tooth root. Particularly, severe edge contact occurred, and micropits and wear occurred from the endface edge to the position of the pit. The gear honing trace was removed due to the wear. So, the wear state is considered as the key point. In order to investigate the state of tooth damage more closely, the tooth flank was measured using a gear tooth measuring machine.

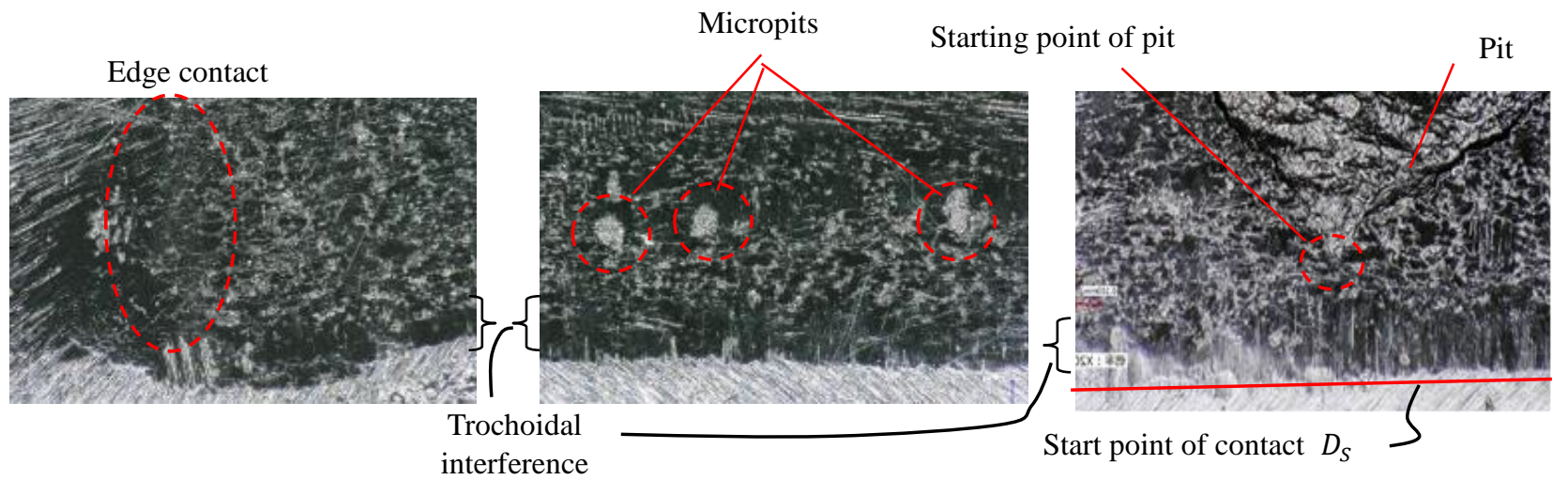

Fig. 15 Enlarged images of tooth root region in Fig. 14. Each image is position described by square figures in Fig. 14. Pits also occurred near the shoulder caused by trochoidal interference on the tooth root. Also, severe edge contact, micropits and wear occurred in pinion A.

Figure 16 shows the tooth flank topography after the durability test. The arrow indicates the gear meshing direction. Since the facewidth of the pinion and driven gear differed, the pinon had a non-contact region on the tooth flank. Figure 17 shows the measured profile of pinion before and after the durability test. It is clear that the concavity due to the trochoidal interference occurred near the start point of meshing toward tooth tip. The position of the shoulder due to the concavity were equivalent to the start point of the pit. Pinion A showed tooth root concavity presumably caused by trochoidal interference due to deflection of the gear teeth under high load. In addition, wear occurred from the endface edge to the center of the facewidth. As a result of the investigation, it is clear that the trochoidal interference extended beyond the base circle could be avoided by the large amount of tooth tip modification of gears, but the shoulder due to trochoidal interference and wear in the lead direction still occurred in pinion A. Therefore, the onset of the shoulder caused by trochoidal interference and wear should be considered more than trochoidal interference extending beyond the base circle. It is considered as a particular phenomenon of high hardness material that the position of pits and the pitting durability are affected by not only the shoulder due to trochoidal interference but also the change in the tooth surface topography caused by wear in the lead direction under high load operating conditions. 


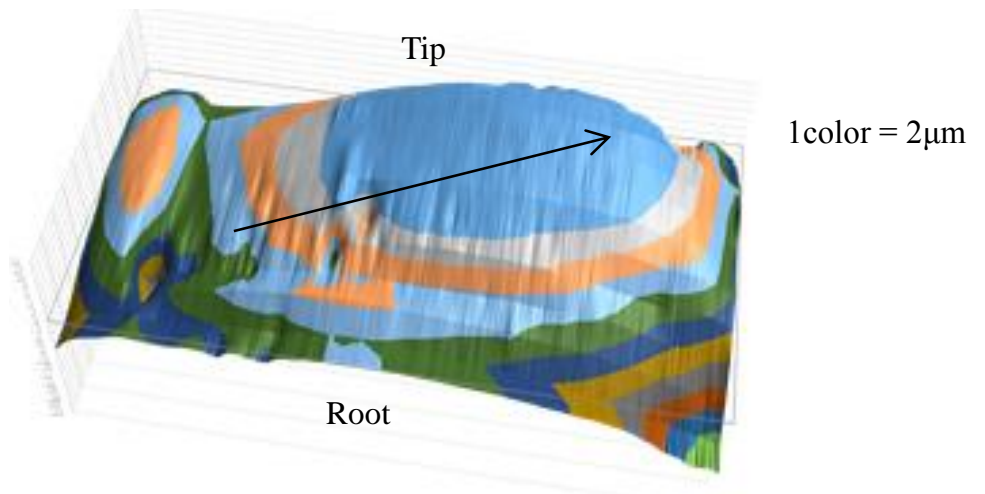

Pinion A

Fig. 16 Tooth surface shape after the durability test. The arrow indicates gear meshing direction. It is clear wear occurred from the endface edge to the center of the facewidth.

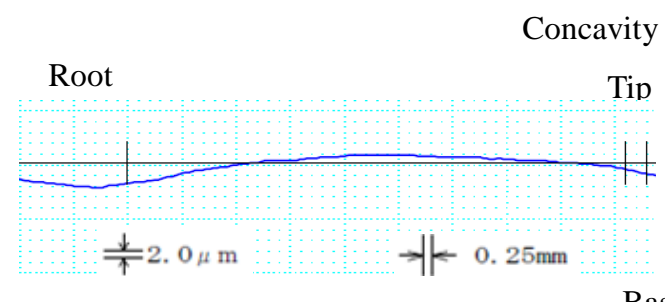

(a) Pinion A, before test

Base circle

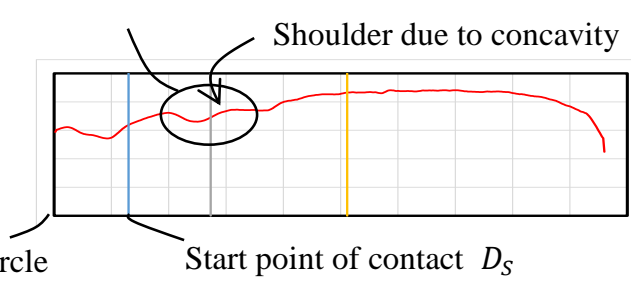

(b) Pinion A, after test

Fig. 17 Measured profile of pinion A before and after durability test. (a) is for averaged profile. (b) is for representative profile.

Observation of tooth surface topography suggested that the severe state of the endface edge contact caused the start point of wear on pinion A because it exceeded the criterion for the onset of wear. Furthermore, wear progressed from the endface edge to the center of the facewidth due to high pressure and sliding friction in the profile direction between engaging gear teeth. The increased pressure promoted the progress of wear near the center of the facewidth of pinion A due to the contact length reduction. In addition, the shoulder on the tooth root due to trochoidal interference led to greater contact stress compositely. As a result, the location of pits changed, i. e., it occurred near the center of the facewidth. As for pitting durability, there are probably cases where the progress of wear may restrain crack propagation that would otherwise develop into pitting, although pitting durability may worsen owing to changes in contact stress or tooth surface roughness.

Figure 18 presents a schematic diagram of the mechanism assumed to cause the change in the location of pits due to wear in the lead direction. Fig. 18-(a) is a replica photo of the entire tooth surface of pinion A in Fig. 14(a). Figure 18(b) shows the tooth profile error at each position in the tooth trace direction. Figure 18-(a) schematically illustrates the presumed mechanism of the relationship between pitting initiation and wear propagation. Generally, pitting due to trochoidal interference presumably starts from a gouge edge crack that propagates to cause pitting. However, at the endface edge in Fig. 18-(b-1), crack propagation would be suppressed by wear that progressed earlier in the lead direction. At the position of Fig. 18-(b-2), although crack and wear would progress at a similar rate, crack propagation was probably suppressed due to wear in the lead direction, so pitting was prevented. In contrast, at the position of Figure 18-(b-3), a crack formed before wear propagation from endface edge to this location and grew to cause pitting. The durability test results for gear pair A showed that micropits were generated at the position of Fig. 18-(b-2), but they did not develop to become large pits. At the position of Fig. 18-(b-3), pits occurred, which presumably means that crack propagation was not suppressed by wear in the lead direction. The interaction between tooth wear in the lead direction and occurrence of trochoidal interference probably caused a change in the location of pits and pitting durability. 
(a)
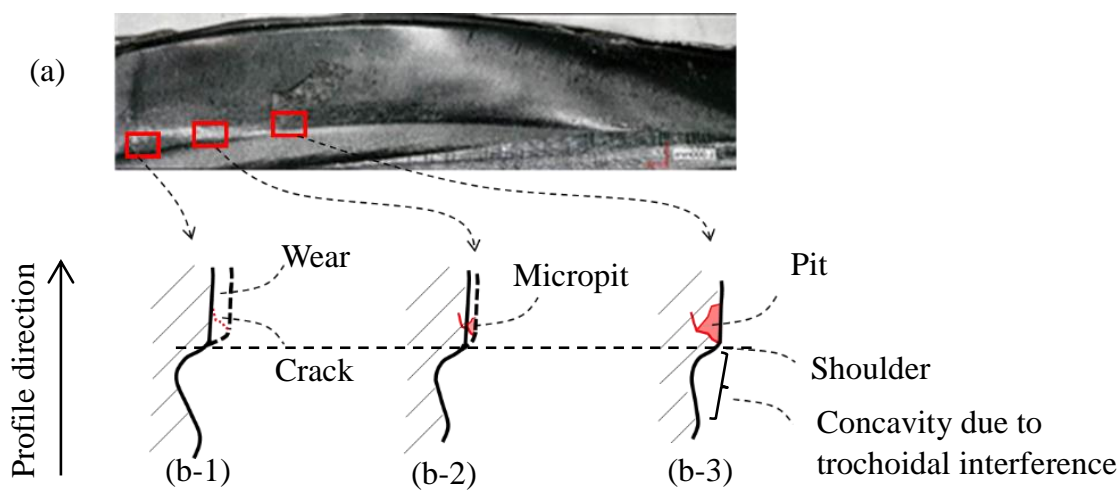

Fig. 18 Schematic diagram of mechanism assumption of competition between wear and pitting.

Thus, the results revealed a new viewpoint that the mechanism causing the variation in the location of pits and pitting durability can be explained by trochoidal interference and multidirectional, interacting wear.

\section{4-2. Verification of the estimated pitting mechanism}

Based on this estimated pitting mechanism, it was assumed that the location of pits and pitting durability will vary if there is a change in the progress of wear in the lead direction. To verify this assumption, a gear durability test was conducted using gear pair B that had the same tooth tip relief as gear pair A but a different endface shape in the lead direction.

Figure 19 shows the measured six diagonal edge shapes of the endface of the driven gear. The edge of gear B was relieved by $50-100 \mu \mathrm{m}$.
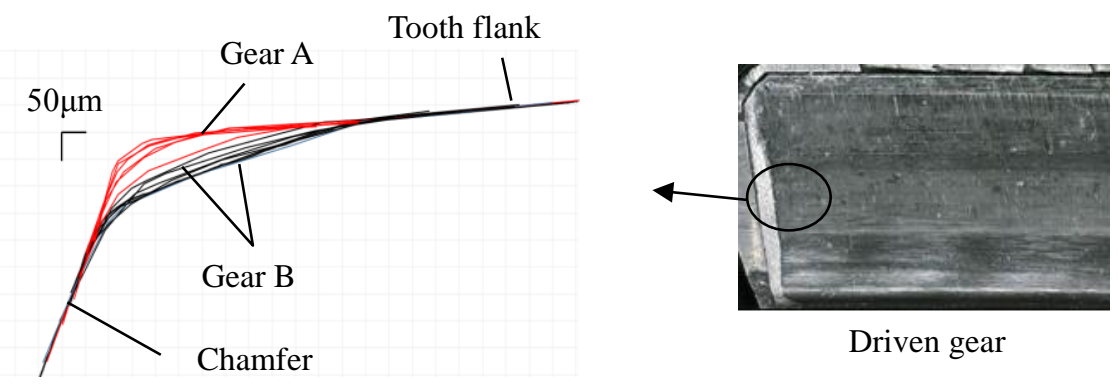

Fig. 19 Different endface edge shapes of gears A and B. There are six diagonal edge shapes, respectively. The edge of gear B was relieved by $50-100 \mu \mathrm{m}$. The red lines and black lines indicate the edge of gear A and gear B, respectively.

As a result of the durability test, the number of load cycles of gear pair B was $1.4 \times 10^{7}$. Photos of the tooth damage on pinion B are shown in Fig. 20. A pit on pinion B was located at the endface edge where gear meshing began. Figure 21 shows an enlarged image of the tooth root region on pinion B in Fig. 20. Trochoidal interference was avoided similar

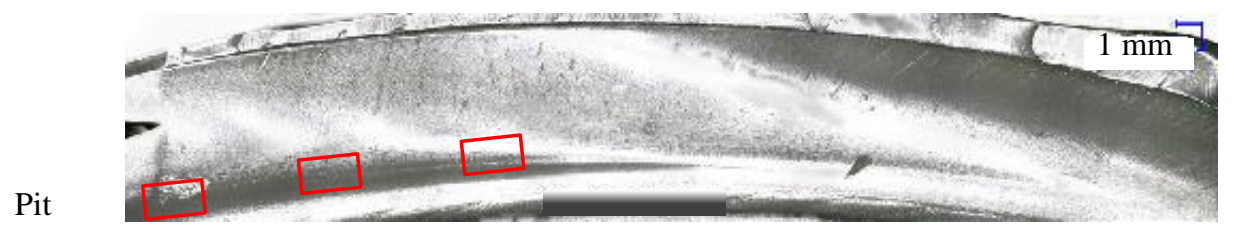

Pinion B, $1.4 \times 10^{7}$ cycles

Fig. 20 State of tooth damage after durability test in the case of gear pair B. The square figures in red refer to Fig. 21.

to pinion A. However, wear did not occur from the endface edge. The gear honing trace remained. In the case of pinion $\mathrm{B}$, owing to the shape of the endface edge relief, wear did not progress like that on pinion A because the criterion for the 
onset of wear was probably not exceeded and the state of contact stress did not become so severe. As a result, the number of load cycles was greater than that of pinion A and the location of pit occurred around the endface as expected.

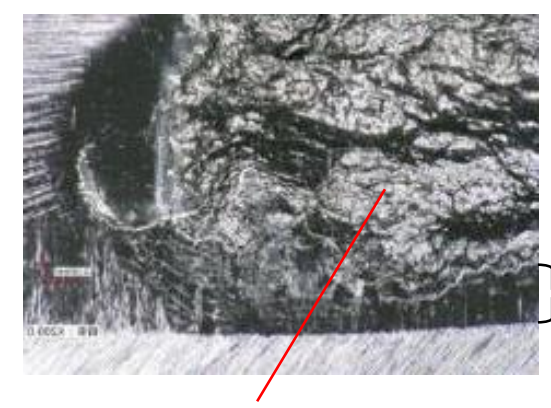

Pit

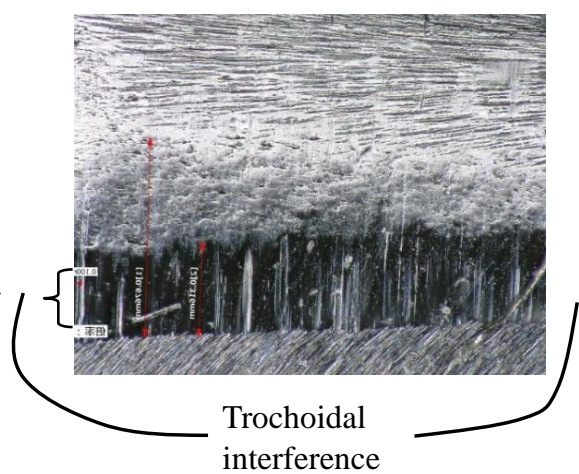

interference

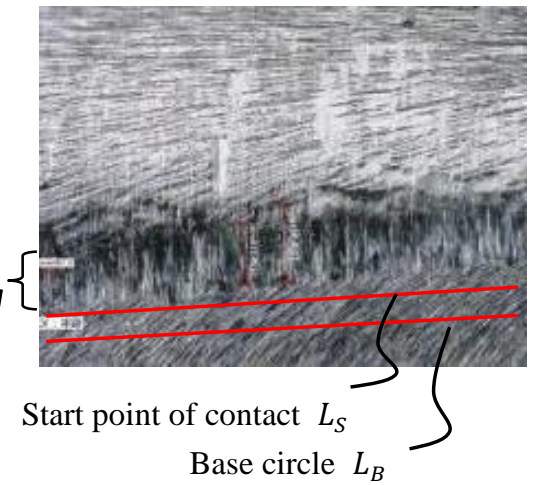

Base circle $L_{B}$

Fig. 21 Enlarged images of tooth root region in Fig. 20. Each image is the position indicated by the squares in Fig. 20. Pit occurred in the meshing starting region and near the shoulder caused by trochoidal interference on the tooth root.

Wear did not occur like that seen for Pinion A.

Figure 22 compares the measured helix before and after the durability tests between pinion A and B. It is also clear that severe wear in the lead direction was avoided on pinion B in the durability test. The results confirmed that the location of pits and the pitting durability changed according to the change in the state of wear in the lead direction.

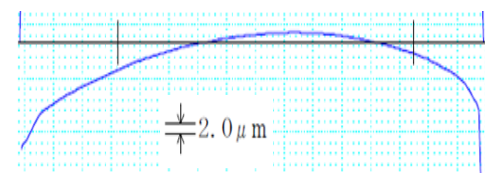

(a) Pinion A, before test

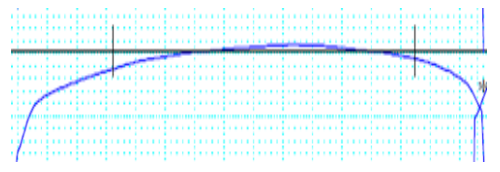

(c) Pinion B, before test

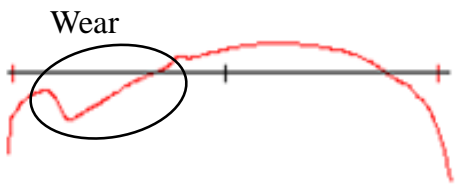

(b) Pinion A, after test

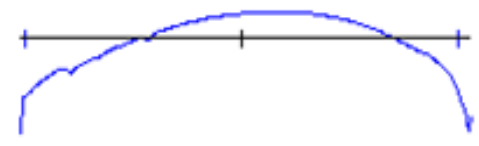

(d) Pinion B, after test

Fig. 22 Measured helix before and after the durability tests. It is also clear that severe wear occurred on pinion A. The boarder of the wear region is equivalent to the position of the pits.

In this case, pitting durability was improved by the endface relief. However, there are probably cases where the progress of wear may restrain crack propagation that would otherwise develop into pitting, although pitting durability may worsen owing to changes in contact stress or tooth surface roughness. The relationship between wear and pitting durability requires further close examination.

The results revealed that pitting durability and the location of pits are influenced sensitively and compositely by not only trochoidal interference but also multidirectional, interacting wear. When the state of the contact exceeded the criterion of pitting earlier than that of wear, pits occurred. Therefore, it is important to consider the influence of trochoidal interference and tooth wear on variation in the meshing state in order to predict pitting durability accurately.

\section{Conclusions}

The following conclusions were obtained from the results of this study.

a) When the gear meshing region is close to the base circle of the pinion, trochoidal interference of the tooth tip of the gear reaches the tooth root region beyond the meshing start region calculated geometrically and extends beyond the base circle. 
b) The concavity due to trochoidal interference near the base circle is suppressed by the tooth tip modification. However, only the modification in the profile direction is not sufficient in order to improve pitting durability.

c) Pitting durability and the location of pits are influenced sensitively and compositely under a high load condition by multidirectional, interacting wear both in profile and lead directions, i.e., not only concavity due to trochoidal interference in the profile direction occurred as expected, but also changes in the location of pits and pitting durability were caused by tooth wear in the lead direction.

d) It is shown that pitting durability and the location of pits are controlled by not only tip modification but also endface modification of gear surface.

\section{References}

Komori, M., Kubo, A., Takahashi, T., Tanaka, T., Ichihara, Y., Takeda, N., Failures of Involute Gears due to Contact of Side Edge and Tip Edge of Tooth, $4^{\text {th }}$ Report, Failure Caused by Trochoidal Interference due to Elastic Deformation of Tooth, Transactions of the JSME C (in Japanese), Vol. 70, No. 700 (2004), pp. 219-227.

Kubo, A. and Umezawa, K., On the power transmitting characteristics of helical gears with manufacturing and alignment errors, $1^{\text {st }}$ Report, Fundamental consideration, Transactions of the JSME (in Japanese), Vol. 43, No. 371 (1977), pp. 2771-2779.

Kubo, A., et al., Gear Failure -Encyclopedia- (2019), pp. 63-74 (in Japanese), Research Institute for Applied Sciences.

Kumagai, K. Morikawa, K. and Takaki, K., A STUDY ON MESH FRICTION LOSS REDUCTION OF CYCLINDRICAL GEARS UNDER MIXED LUBRICATION CONDITION, Proceedings of MPT2017, $07-02$.

Morikawa, K. Nishihara, R. and Matsumoto, S., Estimation and improvement method of mesh efficiency of cylindrical gears, Transactions of the JSME C (in Japanese), Vol. 78, No. 788 (2012), pp. 225-234.

Niemann, G., VDI-Zeitschrift Vol.102 No.6 (1968), pp. 216-224.

Okamoto, D. Ishibasi, M. Matsumoto, M. and Moriguchi, N., Study on Reduction of Gear Loss by Surface Properties, Proceedings of the JSME 2017 (in Japanese), No.17-1, S1120201.

Okumura, T. Morikawa, K. Kumagai, K. and Hosono, K., A Study on Mesh Efficiency of Asymmetric Tooth Gears, Proceedings of the JSME 2017 (in Japanese), No.17-1, S1120202.

Yoshizaki, M., Influence of Trochoidal Interference of Helical Gears on Tooth Surface Strength, Effect of Helix deviation, Crowning and Tip Relief, Transactions of the JSME (in Japanese), Vol. 82, No. 842 (2016), DOI: 10.1299/transjsme.1600288 